\title{
Toward Nanometer-Scale Optical Photolithography: Utilizing the Near-Field of Bowtie Optical Nanoantennas
}

\author{
Arvind Sundaramurthy ${ }^{*}, \dagger, \ddagger$ and Gordon S. Kino ${ }^{\ddagger}$ \\ E.L.Ginzton Laboratory, Stanford University, Stanford, California 94305 \\ P. James Schuck ${ }^{\star}, \dagger, \S$, Nicholas R. Conley $\S$, David P. Fromm $\S$, and W. E. Moerner $\S$ \\ Department of Chemistry, Stanford University, Stanford, California 94305
}

\begin{abstract}
Optically resonant metallic bowtie nanoantennas are utilized as fabrication tools for the first time, resulting in the production of polymer resist nanostructures $<30 \mathrm{~nm}$ in diameter at record low incident multiphoton energy densities. The nanofabrication is accomplished via nonlinear photopolymerization, which is initiated by the enhanced, confined optical fields surrounding the nanoantenna. The position, size, and shape of the resist nanostructures directly correlate with rigorous finite-difference time-domain computations of the field distribution, providing a nanometer-scale measurement of the actual field confinement offered by single optical nanoantennas. In addition, the size of the photoresist regions yields strong upper bounds on photoacid diffusion and resist resolution in SU-8, demonstrating a technique that can be generalized to the study of many current and yet-tobe-developed photoresist systems.
\end{abstract}

\begin{abstract}
The ever-increasing push toward fabrication of ultrasmall features on integrated chips drives much of modern nanotechnology. A prerequisite for nanolithography is the ability to expose and develop photoresist on the scale of a few tens of nanometers. The advent of single optical nanoantennas ${ }^{1-10}$ provides a method for producing ultrasmall features in resist. Optical nanoantennas are specifically engineered to produce greatly enhanced fields at visible and nearinfrared (NIR) wavelengths and to confine them to regions $\sim 20 \mathrm{~nm}$ in size, significantly defeating conventional diffraction limits. Here we fabricate ultrasmall photoresist nanodots using two-photon polymerization enabled by the huge localized enhancement in the vicinity of lithographically produced Au bowtie nanoantennas. Record-low voxel diameters $<30 \mathrm{~nm}$ are generated at record low incident infrared average powers of $27 \mu \mathrm{W}\left(30 \mathrm{~kW} / \mathrm{cm}^{2}\right)$. While sub-diffraction-limited (SDL) resolution has been the goal of near-field optics for some time, 11 nanoantennas comprised of plasmonically coupled metallic nanoparticles offer appreciable advantages over other near-field probes, most notably aperture-based ${ }^{12,13}$ and apertureless ${ }^{1 \text {, }}$ 14,15 near-field scanning optical microscopes (NSOM and ANSOM, respectively).
\end{abstract}

Because single metallic nanoantennas are quite sensitive to their local environment, nonperturbative measurements of the near-field distribution have proven to be difficult. ${ }^{15-19}$ On the other hand, methods involving light-induced changes in polymer matrices are minimally invasive and have been used in the past to characterize scattered optical fields by both one-

\footnotetext{
*Corresponding authors. E-mail: arvisun@stanford.edu, pjschuck@stanford.edu.

†These authors contributed equally to this work.

\$E.L.Ginzton Laboratory.

$\S$ Department of Chemistry.
}

Supporting Information Available: Expanded discussion and interpretation of the wavelength-dependent FDTD simulation results and their correlation with our experimental findings. This material is available free of charge via the Internet at http://pubs.acs.org. 
photon and two-photon absorption. For example, SDL optical resist exposure in the linear regime has been demonstrated (with minimum feature sizes down to $\sim 50 \mathrm{~nm}$ ) for techniques ranging from $\mathrm{NSOM}^{12,20}$ and near-field lithography mask exposure, ${ }^{21}$ to plasmon-assisted exposure. ${ }^{22}$ Similarly, light-generated topographical changes in an azobenzene dye polymer have been employed for mapping near-field distributions, but only for aperture-based systems ${ }^{23}$ and large antenna arrays. ${ }^{24}$ Bowtie nanoantennas pumped on resonance ${ }^{4}$ with a diffraction-limited spot provide a powerful geometry for SDL fabrication. This is a result of their much greater near-field light coupling efficiency than NSOM probes (efficiency $\sim 10 \%$ for a bowtie ${ }^{4}$ versus efficiency $\sim 10^{-5}$ for a typical metal-coated pulled fiber probe ${ }^{12,25}$ where the efficiency of the bowtie antenna is defined as the ratio of the bowtie scattering cross-section area to the area of the diffraction limited excitation spot). In addition, the bowties provide significantly higher field enhancement than has been measured for apertureless tips. $3,26,27$

Two-photon polymerization (TPP) of photoresist, where a two-photon absorption event initiates a cross-linking chain reaction, is an established method for fabricating two- and threedimensional (3D) structures with SDL resolution. ${ }^{28-35}$ Due to the nonlinear dependence of the polymerization on electromagnetic field intensity, exposure occurs only where field strength is greatest, and the fabrication of SDL objects has been demonstrated by both conventional ${ }^{29,31-33,36}$ and tip-enhanced microscopy. ${ }^{30}$ Much of the TPP work in the literature has concentrated on the commercially available epoxy-based photoresist SU-8, which has been optimized for high-aspect-ratio structure fabrication and is well-suited for 3D lithography. ${ }^{29}$ Recently, minimum feature sizes of $\sim 70 \mathrm{~nm}$ and $\sim 30 \mathrm{~nm}$ have been demonstrated for $\mathrm{ANSOM}^{30}$ and confocal-scanning excitation ${ }^{32}$ techniques, respectively, the latter for a specialized structure. We note that while the absorption of wavelengths near $800 \mathrm{~nm}$ in SU-8 has traditionally been described as a two-photon process and is referred to as such here, it is possible that exposure is partially due to a higher-order process.

Our samples consisted of arrays of single bowtie antennas, with bowtie gap widths ranging from 16 to $40 \mathrm{~nm}$, fabricated by e-beam lithography on a fused silica/indium tin oxide (ITO) substrate, as previously reported. ${ }^{2}$ Each triangle of a bowtie was $80 \pm 5 \mathrm{~nm}$ in length. The bowties, spaced $3 \mu \mathrm{m}$ apart, were made of a $20 \mathrm{~nm}$ gold film on a $4 \mathrm{~nm}$ titanium sticking layer. The commercial ultraviolet photoresist SU-8 2002 precursor solution was diluted in a cyclopentanone solvent to $2.5 \mathrm{vol} \%$. Before resist application, samples were cleaned in $\mathrm{KOH} /$ EtOH solution, then rinsed with Nanopure water (EASYpure, resistivity $=18.3 \mathrm{M} \Omega \mathrm{cm}$ ), and dried with a stream of air. Samples were spin coated with a $75 \mathrm{~nm}$ film of SU-8 resist and were baked at 90 and $110^{\circ} \mathrm{C}$ for 2 min each. After exposure, the development process consisted of a 1 min postexposure bake (PEB) at $105^{\circ} \mathrm{C}$, a 1 min cyclopentanone dip to remove the unexposed resist, a 2-propanol rinse, and drying with a heat gun. All samples were then subjected to a $\sim 5$ min UV hard bake with an Hg lamp.

The exposures were performed in a sample-scanning inverted optical microscope. The excitation source for the exposure was a mode-locked Ti:sapphire laser (pulse width $\tau=120$ fs, repetition rate $f=75 \mathrm{MHz}$ and wavelength $\lambda_{\text {incident }}=800 \mathrm{~nm}$ ). The $800 \mathrm{~nm}$ wavelength was chosen to ensure sufficient TPA in the resist and to excite the bowtie antennas reasonably close to resonance. ${ }^{4}$ The laser was focused to a diffraction-limited spot at the sample by a 1.3 numerical aperture (NA) $100 \times$ oil objective. The focal plane position on the sample was confirmed by a charge-coupled device (CCD) camera attached to the front port of the microscope. The samples were exposed at average powers ranging from $27 \mu \mathrm{W}$ to $10 \mathrm{~mW}$. A $20 \mu \mathrm{m} \times 20 \mu \mathrm{m}$ area containing at least 36 bowties was scanned at each power level. Each scan consisted of $200 \times 200$ pixels $(100 \mathrm{~nm} /$ pixel) with a $10 \mathrm{~ms}$ dwell time/pixel. Two-photonexcited photoluminescence (TPPL) from the bowties was simultaneously collected with the same objective (as previously described ${ }^{3}$ ) during the exposure and provided a direct experimental measurement of the field enhancement at every scanned bowtie. We have 
separately determined that neither the TPPL emission nor any second harmonic signal from the bowtie is responsible for the photopolymerization.

An atomic force microscope (tapping AFM, Si cantilever tip, Digital Instruments 3000) image of developed regions exposed with 4.7 and $2.3 \mathrm{~mW}$ average laser powers is shown in Figure 1a. At these high powers, TPP occurs in the absence of the enhanced bowtie fields and hence we see a continuous layer of exposed resist in the AFM image. In addition to the exposed resist layer, we also observe holes over areas where the bowties were present. At these high incident powers, the enhanced fields at the bowties were enough to ablate ${ }^{37}$ or thermally boil-off ${ }^{29}$ the resist around the bowties, thus providing initial proof of field enhancement in the nanoantennas. The high incident powers (average powers $\geq 500 \mu \mathrm{W}$ ) also result in bowtie damage. We note that the TPP threshold average power in the absence of bowties was found to be $1.8 \mathrm{~mW}$ for our particular film thickness, step size, and dwell time, consistent with the threshold found in previous studies. ${ }^{29,32}$

Figure $1 \mathrm{~b}$ is a scanning electron microscope (SEM) image of the boundary region between a $2.3 \mathrm{~mW}$ scan, a $1.0 \mathrm{~mW}$ scan, and an unexposed region (to the right of the dotted line in Figure 1b). In the SEM, the efficiency of secondary electron emission is much higher for conductors than for insulators. This, coupled with a small penetration depth associated with the low energy primary electron beam $(2 \mathrm{KV})$, makes the SU-8 resist layer appear dark in contrast to the bright gold and ITO regions. This becomes apparent in Figure 1b, where blanket TPP occurs at the $2.3 \mathrm{~mW}$ incident power. As the power is reduced, however, TPP is observed only near the bowties, which is demonstrated by the slightly darkened areas surrounding the bowties in the $1 \mathrm{~mW}$ scan region of Figure $1 \mathrm{~b}$. To better see what happens at low powers, parts $\mathrm{c}$ and $\mathrm{d}$ of Figure 1 show SEM images of a bowtie antenna exposed at an average power of $120 \mu \mathrm{W}$ and an unexposed antenna, both of which have gap sizes of $\sim 36 \mathrm{~nm}$. For the $120 \mu \mathrm{W}$ exposure, we observe a dark region primarily confined near the bowtie gap, in contrast to the unexposed bowtie. This provides experimental validation of resist exposure in the vicinity of the gap between triangles in the antenna and is a signature of strong field confinement. Although the SEM does not show the exact size of the polymer region due to the low acceleration voltage, the partial transparency of the exposed SU-8 in SEM images still allows us to measure the gap sizes of bowties, the importance of which is discussed below.

In addition to SEM imaging, quantitative topographical characterization of resist features on exposed bowties was obtained via atomic force microscopy (AFM). Figure 2a shows an AFM image for a bowtie exposed at $225 \mu \mathrm{W}$. At this incident power, the optical field is strong enough to generate significant amounts of photoacid, and the entire antenna is covered with resist after development (see line scan in the lower part of the figure). The field is strongly enhanced in the vicinity of the gap, resulting in a high concentration of photoacid in this region, and we therefore observe taller resist features near the gap. During the 1 min PEB, these acids diffuse away from the highly concentrated region, ultimately covering the bowtie and surrounding region with cross-linked resist. We also find excess resist exposure at the back center of each triangle in the bowtie. Even though finite-difference time-domain (FDTD) simulations predict slightly enhanced regions at the back corners, ${ }^{6}$ the lack of polymer deposition at the corners could signal excess roughness near the center of the back edge. At a power level of $106 \mu \mathrm{W}$ (Figure 2b) we begin to observe better resist confinement close to the triangle tips near the gap. When the incident power level is further reduced to $54 \mu \mathrm{W}$ (Figure 2c), the observed confinement is even sharper, but the polymer shape produced for this particular bowtie is hard to reconcile precisely with FDTD simulations and may be due to the probabilistic nature of the photoacid diffusion. The lowest power level at which we observed resist exposure was $27 \mu \mathrm{W}$ (Figure 2d). At this power level, two resist pillars located just at the tips of the constituent triangles in the bowtie are observed. The resist height is maximum at the tip of the triangles, indicating that the highest field enhancement occurs here and falls off in the gap. We measured 
a full width half-maximum (fwhm) feature size of $\sim 30 \mathrm{~nm}$ for each of the resist pillars. Also, exposure at this average incident power corresponds to an intensity enhancement of $>67$ at the bowtie surface, determined by computing the ratio of the unenhanced exposure threshold to that for the resist-covered bowtie. Essentially, the bowtie nanoantenna "captures" the energy present in the diffraction-limited excitation spot and concentrates it into two very small areas at the bowtie tips near the gap, ${ }^{4}$ resulting in an increased energy density in these nanoscale regions that exceeds the threshold exposure. These represent the best values for light confinement and enhancement measured experimentally in the near-field of a lithographically fabricated nanoantenna thus far. We note that the raised resist features in the AFM images appear wider than they actually are due to convolution ${ }^{38}$ between the tip (whose radius of curvature is comparable to the dimensions being measured) and the sample. It may therefore be concluded that the features are narrower than $30 \mathrm{~nm}$.

The trends displayed in Figure 2 are indicative of the TPP trends observed for more than 30 bowtie exposures performed at each power. For incident average powers between 100 and 500 $\mu \mathrm{W}$, exposed features are always observed near the gap, at the primary regions of field enhancement. Also at these powers, secondary features are often observed at regions on the bowtie corresponding to points of lesser, but finite, field enhancement (i.e., at edges and the outside corners). At the lowest incident exposure powers, 54 and $27 \mu \mathrm{W}$, exposed resist is confined near the gap and is present only for bowties with gap sizes between 30 and $40 \mathrm{~nm}$. It is important to note that nearly all the features fabricated here are well below $100 \mathrm{~nm}$ in dimension. In this regime, feature size, shape, and position are significantly influenced by the fundamental resolution of the resist, which for a chemically amplified resist like SU-8 is determined by the stochastic and dynamic nature of photoacid diffusion and reaction kinetics. The ultrahigh spatial resolution provided by the bowtie affords us the opportunity to estimate upper bounds for the properties that fundamentally limit lithographic reproducibility, which is discussed in more detail below.

Along with the experimental measurements, we also studied the field enhancement and confinement in bowtie antennas by FDTD simulations, building upon previously reported studies. $^{2,4}$ The FDTD simulations for this work modeled bowtie antennas that closely resembled the fabricated structures covered with a $76 \mathrm{~nm}$ layer of SU-8 resist (the SU-8 optical properties are known at $\lambda_{\text {incident }}=800 \mathrm{~nm}$ ). As expected, the experimentally measured polymer exposure and intensity enhancement from TPPL were strongly correlated. From SEM analysis, we observed that our best resist exposures (at 27 and $54 \mu \mathrm{W}$ ) arose from bowties with $\sim 36 \mathrm{~nm}$ gaps, and FDTD computations for a $36 \mathrm{~nm}$ gap width bowtie are shown in Figure $3 \mathrm{a}$. The FDTD simulations predict an intensity enhancement of 107 at $4 \mathrm{~nm}$ above each of the triangle tips of the resist-covered nanoantenna. This intensity enhancement falls off to $\sim 16$ in the gap between the triangles. As can be seen in Figure 3b, the profile of the resist features for the 27 $\mu \mathrm{W}$ case, a measure of confined field enhancement and distribution, correlates well with the rigorous FDTD simulations of field distribution.

The enhancement values obtained from TPPL measurements for bowties with $\sim 36 \mathrm{~nm}$ gaps were higher than those obtained from bowties with smaller gaps (e.g., gap sizes of $\sim 16 \mathrm{~nm}$ ). This can easily be understood from the fact that the resist-covered bowtie has a different peak resonance wavelength behavior than that for a bare bowtie. For our fixed pumping wavelength of $800 \mathrm{~nm}$ (to ensure sufficient TPP), the resist-covered bowtie with resonance closest to 800 $\mathrm{nm}$ is at a gap of $\sim 40 \mathrm{~nm} .{ }^{4}$ For a $36 \mathrm{~nm}$ gap bowtie, FDTD computations yield a $\lambda_{\text {res }}=872 \mathrm{~nm}$ and an intensity enhancement of $\sim 353$ when pumped on resonance. We note that the intensity enhancement figures obtained independently from the resist exposure (>67) and TPPL measurement $(\sim 150)$ for the bowtie exposed at $27 \mu \mathrm{W}$ (Figure $2 \mathrm{~d}$ ) are roughly consistent, and this is the case in general. Both values also generally agree with intensity enhancement predicted by FDTD $(\sim 107)$ for a bowtie with a $36 \mathrm{~nm}$ gap. ${ }^{4}$ 
Due to the nature of chemically amplified photoresists, nanostructure fabrication via a nanoscale light source is predominantly determined by probabilistic factors, especially near the TPP threshold (e.g., probability of a TPA event occurring, stochastic diffusion, etc.). 39-41 While resist features similar in size and shape to those in Figure $2 \mathrm{~d}$ were fabricated for many bowties, no smaller features were observed on the samples. If this is the minimum feature size attainable in the SU-8 2002 resist system (or is at least the minimum size that adheres to the surface), then an upper bound on the number of cross-links initiated by a single photoacid and on acid diffusion is obtained by assuming the smallest feature results from the generation of a single photoacid. On the basis of the known SU-8 density $\left(\sim 1200 \mathrm{~kg} / \mathrm{m}^{3}\right)$ and molecular weight of the SU-8 monomer (1398 g/mol), each of the two resist features in Figure 2d contain approximately 500 monomers. Since each monomer in a cross-linked region is connected to seven others on average, ${ }^{29}$ then a single photoacid can initiate at most $(500 \times 7) / 2=1750$ crosslinks during the $1 \mathrm{~min}$ PEB (it is worth noting here that no radical quenchers are intentionally introduced to SU-8). Furthermore, the Figure $2 \mathrm{~d}$ resist features are approximately hemispherical in shape with a radius of $\sim 10 \mathrm{~nm}$, yielding an upper bound for photoacid displacement in $1 \mathrm{~min}$ (at $105^{\circ} \mathrm{C}$ ). This movement is determined by both (time-dependent) photoacid diffusion and resist reaction kinetics, ${ }^{39-41}$ and it is unknown which of these is the limiting effect. If one further assumes diffusion-limited movement (and that diffusion occurs for the full $60 \mathrm{~s}$ ), then a diffusion constant upper bound of $3 \times 10^{-14} \mathrm{~cm}^{2} / \mathrm{s}$ is found for this system using the 3D Brownian motion formula: $\left\langle r^{2}\right\rangle=6 D t$ where $r$ is the position of the diffusing particle after time $t$ when measured from its starting position and $D$ is the diffusion constant. This value falls well within the (large) range of photoacid diffusion constants reported in the literature for a number of resist systems. ${ }^{39}$ Certainly, nanoscale fabrication via nonlinear excitation will be improved by tailoring resists for this type of photolithography. ${ }^{31,33,34,36}$ In fact, bowties acting as highly localized light sources are an excellent tool for elucidating and optimizing fundamental resist polymer properties.

The applications for bowties will be further expanded by fabricating them on cantilevers to produce a scanning illumination method. This can be used for scanning probe lithography (SPL) on scales that match the future line width requirements stipulated by the integrated circuit industry. The bowties can also replace apertures on masks to perform high throughput contact optical lithography by two-photon exposure of optical resist, obviating the need for shorter wavelength sources, while still retaining the ability to fabricate isolated small features. The TPP of photoresist by the enhanced fields in a bowtie also provides a mechanism for trapping quantum objects (e.g., single molecules or quantum dots) in a polymer matrix precisely at the location of the "hot spots" of the bowtie. When coupled to the bowtie plasmon resonance, the quantum object will interact directly with the enhanced and localized fields. This improved mismatch between light and nanoscale objects has significant ramifications for ultrasensitive emission and detection applications such as single-photon sources for quantum key distribution $^{42}$ or analyte detection based on spectroscopic changes of the polymer nanodot, respectively.

In conclusion, bowtie nanoantennas concentrate visible and NIR light to regions $\sim 20 \mathrm{~nm}$ in size and therefore provide a unique tool for scientific investigation on these length scales. We have fabricated polymeric nanostructures $<30 \mathrm{~nm}$ in diameter at record low incident power using nonlinear photopolymerization by the enhanced, confined optical fields. In addition, the position, size, and shape of the nanostructures directly correlates with FDTD computations of the field distribution, providing the first nanometer-scale measurement of the actual field confinement offered by optical nanoantennas. The size of the photoresist regions yields strong upper bounds on photoacid diffusion and resist resolution in SU-8, demonstrating a technique that can be generalized to many current and yet-to-be developed photoresist systems. 


\section{Supplementary Material}

Refer to Web version on PubMed Central for supplementary material.

\section{Acknowledgments}

This work was supported in part by the U.S. Department of Energy Grant No. DE-FG03-00ER45815 (GK), the National Institutes of Health Grant No.1R21-GM65331 (WEM), and by the Stanford Nanofabrication Facility (a member of the National Nanotechnology Infrastructure Network) which is supported by the National Science Foundation, Grant ECS-9731293. The authors thank Professor Andy Neureuther, University of California, Berkeley for use of TEMPEST 6.0 FDTD software developed by his group, and Professor Calvin F. Quate for helpful discussions.

\section{References}

1. Sanchez EJ, Novotny L, Xie XS. Phys. Rev. Lett 1999;82:4014-4017.

2. Fromm DP, Sundaramurthy A, Schuck PJ, Kino GS, Moerner WE. Nano Lett 2004;4:957-961.

3. Schuck PJ, Fromm DP, Sundaramurthy A, Kino GS, Moerner WE. Phys. Rev. Lett 2005;94:017402. [PubMed: 15698131]

4. Sundaramurthy A, Crozier KB, Kino GS, Fromm DP, Schuck PJ, Moerner WE. Phys. Rev. B 2005;72:165409.

5. Muhlschlegel P, Eisler H-J, Martin OJF, Hecht B, Pohl DW. Science 2005;308:1607-1609. [PubMed: 15947182]

6. Hao E, Schatz GC. J. Chem. Phys 2004;120:357-366. [PubMed: 15267296]

7. Kottmann JP, Martin OJF, Smith DR, Schultz S. J. Microsc 2001;202:60-65. [PubMed: 11298871]

8. Genov DA, Sarychev AK, Shalaev VM, Wei A. Nano Lett 2003;4:153-158.

9. Jackson JB, Westcott SL, Hirsch LR, West JL, Halas NJ. Appl. Phys. Lett 2003;82:257-259.

10. Farrer RA, Butterfield FL, Chen VW, Fourkas JT. Nano Lett 2005;5:1139-1142. [PubMed: 15943457]

11. Paesler, MA.; Moyer, PJ. Near-Field Optics Theory, Instrumentation, and Applications. Wiley; New York: 1996.

12. Betzig E, Trautman JK, Harris TD, Weiner JS, Kostelak RL. Science 1991;251:146-1470.

13. Hecht B, Sick B, Wild UP, Deckert V, Zenobi R, Martin OJF, Pohl DW. J. Chem. Phys 2000;112:7761-7774.

14. Hamann HF, Gallagher A, Nesbitt DJ. Appl. Phys. Lett 1998;73:1469-1471.

15. Hillenbrand R, Keilmann F. Appl. Phys. Lett 2002;80:25-27.

16. Hillenbrand R, Keilmann F. Appl. Phys. B 2001;73:239-243.

17. Hillenbrand R, Keilmann F, Hanarp P, Sutherland DS, Aizpurua J. Appl. Phys. Lett 2003;83:368370.

18. Leiderer P, Bartels C, Konig-Birk J, Mosbacher M, Boneberg J. Appl. Phys. Lett 2004;85:5370-5372.

19. Bouhelier A, Beversluis MR, Novotny L. Appl. Phys. Lett 2003;83:5041-5043.

20. Aizenberg J, Rogers JA, Paul KE, Whitesides GM. Appl. Phys. Lett 1997;71:3773-3775.

21. Alkaisi MM, Blaikie RJ, McNab SJ, Cheung R, Cumming DRS. Appl. Phys. Lett 1999;75:35603562.

22. Kik PG, Maier SA, Atwater HA. Proc. SPIE 2004;5347:215-223.

23. Davy S, Spajer M. Appl. Phys. Lett 1996;69:3306-3308.

24. Hubert C, Rumyantseva A, Lerondel G, Grand J, Kostcheev S, Billot L, Vial A, Bachelot R, Royer P, Chang S.-h. Gray SK, Wiederrecht GP, Schatz GC. Nano Lett 2005;5:615-619. [PubMed: 15826096]

25. Novotny L, Pohl DW, Hecht B. Opt. Lett 1995;20:970-972. [PubMed: 19859393]

26. Hartschuh A, Beversluis MR, Bouhelier A, Novotny L. Philos. Trans. R. Soc. London, Ser. A 2004;362:807-819.

27. Martin YC, Hamann HF, Wickramasinghe HK. J. Appl. Phys 2001;89:5774-5778.

28. Tanaka T, Sun H-B, Kawata S. Appl. Phys. Lett 2002;80:312-314. 
29. Teh WH, Durig U, Drechsler U, Smith CG, Guntherodt H-J. J. Appl. Phys 2005;97:054907.

30. Yin X, Fang N, Zhang X, Martini IB, Schwartz BJ. Appl. Phys. Lett 2002;81:3663-3665.

31. Baldacchini T, LaFratta CN, Farrer RA, Teich MC, Saleh BEA, Naughton MJ, Fourkas JT. J. Appl. Phys 2004;95:6072-6076.

32. Juodkazis S, Mizeikis V, Seet KK, Miwa M, Misawa H. Nanotechnology 2005;16:846-849.

33. Campagnola PJ, Delguidice DM, Epling GA, Hoffacker KD, Howell AR, Pitts JD, Goodman SL. Macromolecules 2000;33:1511-1513.

34. Cumpston BH, Ananthavel SP, Barlow S, Dyer DL, Ehrlich EE, Erskine LL, Heikal AA, Kuebler SM, Lee I-Y, McCord-Maughon D, Qin J, Rockel H, Rumi M, X.-L. W, Marder SR, Perry JW. Nature 1999;398:51-54.

35. Postnikova BJ, Currie J, Doyle T, E. HR, Anslyn EV, Shear JB, Vanden Bout DA. Microelectron. Eng 2003;69:459-465.

36. Takada K, H.-B. S, Kawata S. Appl. Phys. Lett 2005;86:071122.

37. Perry MD, Stuart BC, Banks PS, Feit MD, Yanovsky V, Rubenchik AM. J. Appl. Phys 1999;85:68036810.

38. Montelius L, Tegenfeldt JO. Appl. Phys. Lett 1993;62:2628-2630.

39. Houle FA, Hinsberg WD, Morrison M, Sanchez MI, Wallraff G, Larson C, Hoffnagle J. J. Vac. Sci. Technol., B 2000;18:1874-1885.

40. Lin EK, Soles CL, Goldfarb DL, Trinque BC, Burns SD, Jones RL, Lenhart JL, Angelopoulos M, Willson CG, Satija SK, Wu W-L. Science 2002;297:372-375. [PubMed: 12130778]

41. Mason MD, Ray K, Grober RD, Pohlers G, Cameron J. Phys. Rev. Lett 2004;93:073004. [PubMed: 15324233]

42. Lounis B, Moerner WE. Nature 2000;407:491-493. [PubMed: 11028995] 

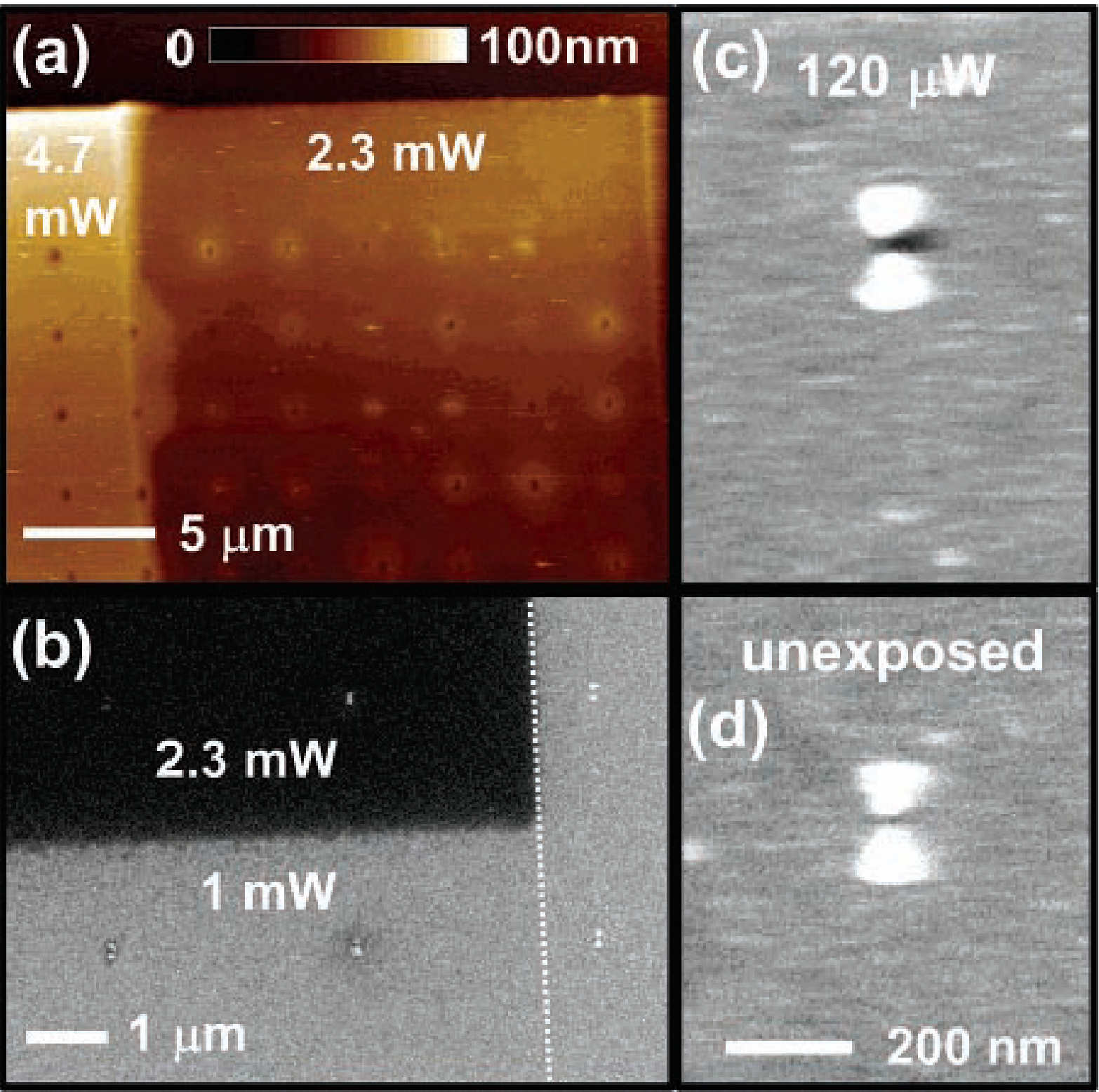

Figure 1.

(a) AFM image showing large-scale resist exposure at average powers of 4.7 and $2.3 \mathrm{~mW}$. The holes coincide with the presence of bowties in the array. (b) SEM image of the boundary region between a $2.3 \mathrm{~mW}$ scan, a $1.0 \mathrm{~mW}$ scan, and an unexposed region (to the right of the dotted line). We observe blanket TPP for the $2.3 \mathrm{~mW}$ scan but exposure only near bowties for the 1 $\mathrm{mW}$ scan (note dark areas around bowties in this region). All exposed bowties in (a) and (b) were damaged by the high excitation fields. (c) SEM image of bowtie (36 nm gap) exposed at $120 \mu \mathrm{W}$ showing a dark, unresolved spot from exposed photoresist and (d) an unexposed bowtie. 
(a)

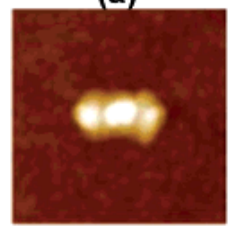

(b)

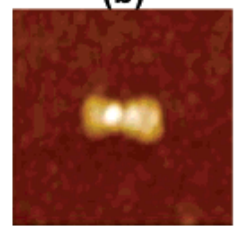

(c)

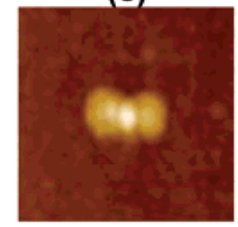

(d)

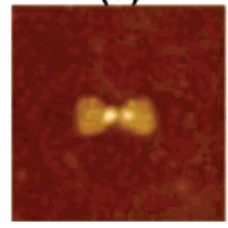

(e)
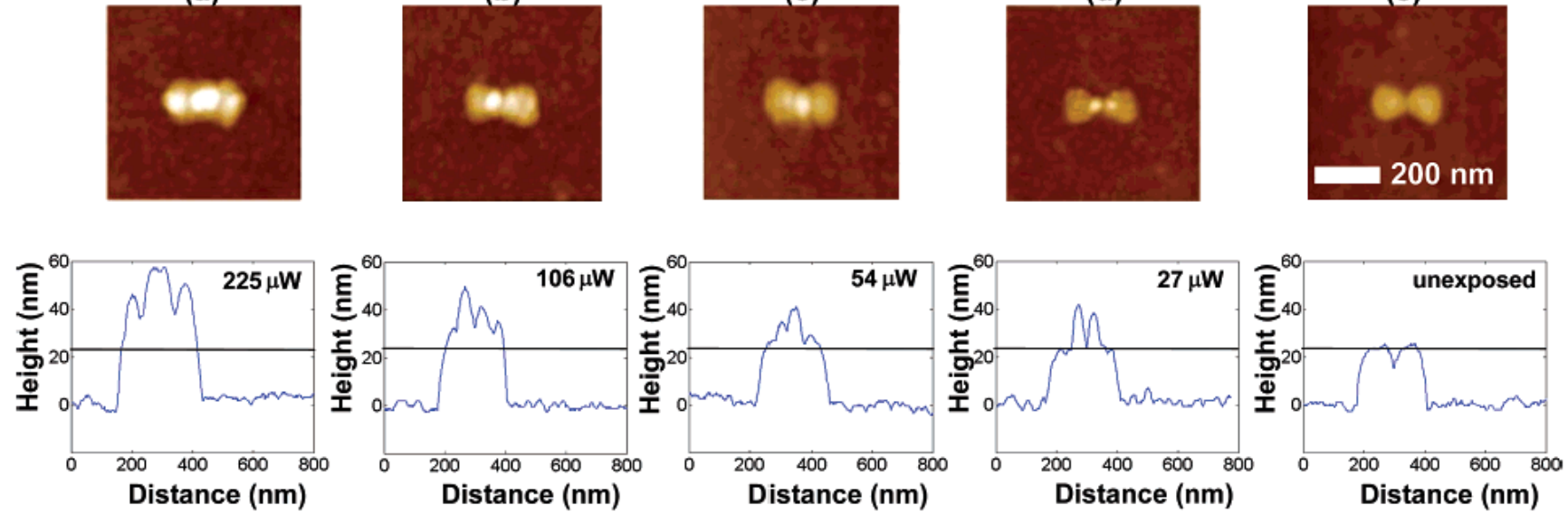

Figure 2.

AFM images and cross sections along the nanoantenna axis of bowties exposed at (a) 225 $\mu \mathrm{W}$, (b) $106 \mu \mathrm{W}$, (c) $54 \mu \mathrm{W}$, (d) a record low $27 \mu \mathrm{W}$, and (e) unexposed bowtie. The black line in the cross-section figures shows the bowtie height before exposure. Each of these bowties has a gap size of $\sim 36 \mathrm{~nm}$. 


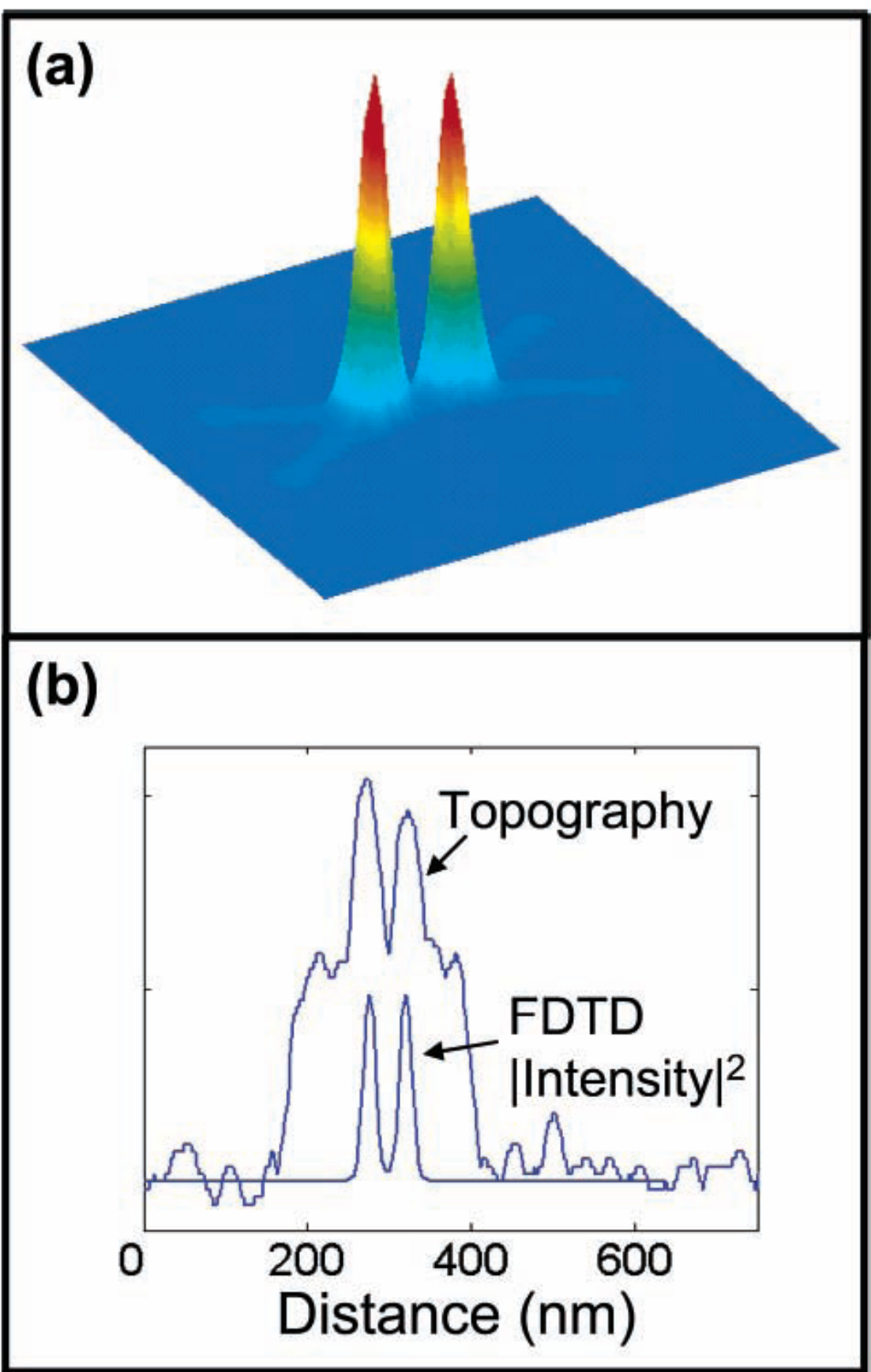

Figure 3.

(a) Color surface plot of FDTD-calculated $|I|^{2}\left(|E|^{4}\right)$ distribution in plane parallel to the surface $4 \mathrm{~nm}$ above a| bowtie with a $36 \mathrm{~nm}$ gap. The peak $|I|^{2}\left(|E|^{4}\right)$ enhancement value for the FDTD plot is 11450 and the minimum value is 1 . The squared intensity is plotted since TPP is the result of a two-photon absorption process. (b) Comparison of the resist exposure topographical cross section obtained at $27 \mu \mathrm{W}$ and a linecut through the bowtie axis of the $|I|^{2}$ plot obtained from FDTD simulations. The peak positions of the resist topography and $|I|^{2}$ field distributions agree to within one FDTD simulation node (4 $\mathrm{nm})$. 